\title{
ARTÍCULOS
}

Recibido 29.10.2012. Aprobado 03.09.2013

Evaluado por el sistema double blind review. Editor Científico: Eduardo Ayrosa

DOI: http://dx.doi.org/10.1590/So034-759020140405

\section{SATISFACCIÓN: DETERMINANTE DE LA FAMILIARIDAD DEL DESTINO TURÍSTICO}

\author{
Satisfação: determinante da familiaridade do destino turístico \\ Satisfaction: the determinant of tourist destination familiarity
}

\begin{abstract}
RESUMEN
Este estudio pretende probar que la satisfacción obtenida por el turista, es consecuencia del beneficio funcional, hedónico y simbólico adquirido en el lugar. Y a su vez, probar si la satisfacción es un antecedente, para que el destino turístico le sea más familiar. A través de un análisis factorial confirmatorio (AFC), se identificaron diferentes escalas que permitieron medir dichas relaciones. Se utilizó un muestreo no probabilístico con base en cuotas, en proporción a destinos turísticos visitados en Chile. La relación entre la percepción de beneficios como antecedente de la satisfacción, puede ser muy importante para que el destino turístico sea percibido como más familiar que otros. Este estudio sugiere que la satisfacción cumple un rol mediador muy importante, entre la percepción de beneficios y la familiaridad del destino turístico. Las autoridades deberían considerar la triada de beneficios como base para establecer estrategias de marketing que busquen potenciar la satisfacción.
\end{abstract}

PALABRAS CLAVES / Familiaridad, satisfacción, mediación, beneficios, turista.

\section{RESUMO}

Este estudo pretende provar que a satisfação obtida pelo turista, é consequência do benefício funcional, hedônico e simbólico adquirido no local. E por sua vez, provar sim a satisfação é um antecedente, para que o destino turístico se torne mais familiar. Através de uma análise fatorial confirmatória (AFC), são identificadas diferentes escalas que permitiram medir estas relações. Uma amostra não probabilística foi utilizada com base nas cotas, na proporção dos destinos turísticos visitados no Chile. A relação entre a percepção dos benefícios como antecedente da satisfação pode ser muito importante para que o destino turístico seja percebido como mais familiar que outros. Este estudo sugere que a satisfação cumpre com uma função mediadora muito importante, entre a percepção dos benefícios $e$ a familiaridade do destino turístico. As autoridades deveriam considerar a tríade de benefícios como base para estabelecer estratégias de marketing que busquem aumentar a satisfação.

\section{EDUARDO TORRES MORAGA}

eduardot@unegocios.cl

Profesor de la Facultad de Economía

y Negocios, Universidad de Chile,

Santiago - Chile.

\section{CORO CHASCO YRIGOYEN}

coro.chasco@uam.es

Profesora de la Facultad de Economía y Negocios, Universidad Autónoma de Madrid, Madrid - España.

\section{enrique.marinao@usach.cl Profesor de la Facultad de Administración y Economía, Universidad de Santiago de Chile, Santiago - Chile. \\ ENRIQUE MARINAO ARTIGAS}

PALAVRAS-CHAVE / Familiaridade, satisfação, mediação, benefícios, turista.

\begin{abstract}
This study aims to prove the satisfaction felt by a tourist is the result of the functional, hedonic and symbolic benefits acquired from the site. In its turn, it aims to prove that satisfaction is an antecedent for the tourist destination to become more familiar. Different scales are identified to permit measuring these relations based on a confirmatory factor analysis (CFA). A non-probabilistic sample was used based on quotas, in the proportion of tourist destinations visited in Chile. The relationship between perceiving benefits as antecedents for satisfaction can be very important for the tourist destination to be perceived as more familiar than other destinations. This study suggests that satisfaction complies with a very important mediator function between the perception of benefits and the familiarity of the tourist destination. Authorities should consider the triad of benefits as a basis for establishing marketing strategies that seek an increase in satisfaction.
\end{abstract}

KEY WORDS / Familiarity, satisfaction, mediation, benefits, tourist. 


\section{INTRODUCCIÓN}

La satisfacción de los clientes conduce al éxito de la industria turística (Stevens, Knutson, \& Patton, 1995) y de los destinos turísticos (Pizam, Neumann, \& Reichel, 1978). La satisfacción ha sido uno de los componentes que más se ha estudiado inherentes al comportamiento del turista. Por un lado, como antecedente del comportamiento (Yüksel, Yüksel, \& Bilim, 2010) y por otro, como consecuencia (Lee, Lee, \& Choi, 2011). Para un turista que experimente satisfacción en el lugar, le será más fácil recomendarlo o regresar (Chen \& Chen, 2010; Lee, Lee \& Choi, 2011) y expresar lealtad hacia él (Yüksel, Yüksel \& Bilim, 2010).

La familiaridad es una de las dimensiones que cumple un importante rol en la elección (Gefen \& Straub, 2004) que el turista realiza para visitar un lugar. Puede influir en la imagen del destino (Prentice, 2004), en el apego del turista hacia el lugar (Hammitt, Kyle, \& Oh, 2009). Es un antecedente del placer que experimente el turista (Andsager \& Drzwiecka, 2002), lo que podría generar relaciones a largo plazo y estimular su regreso (Tsai, 2012), generando actitudes de lealtad hacia el lugar.

La satisfacción del turista es una consecuencia de la calidad de los servicios recibidos (Chen \& Chen, 2010), de la imagen percibida (Bigné, Sánchez, \& Sánchez, 2001), del placer emocional (De Rojas \& Camero, 2008) y del valor percibido (Chen \& Chen, 2010) en el lugar. Es el resultado de la variedad de atributos con los que cuenta el destino turístico y de las percepciones del turista (Hui, Wan, \& Ho, 2007). Los beneficios obtenidos, son factores que determinan la satisfacción (Lam, Shankar, Krishna, \& Murthy, 2004) del turista. La satisfacción cumple un rol mediador muy importante entre la percepción de beneficios y la familiaridad. No obstante, la literatura especializada entrega escasa evidencia de ese rol. En este estudio se propone un modelo en el que la satisfacción es un antecedente de la familiaridad del destino turístico y una consecuencia de los beneficios funcionales, hedónicos y simbólicos, percibidos por el turista en el lugar.

\section{REVISIÓN DE LA LITERATURA}

Con el fin de dar a este estudio una aceptable fuerza teórica, a continuación se presenta una exhaustiva revisión de la literatura de distintas fuentes bibliográficas.

\section{PERCEPCIÓN DE BENEFICIO FUNCIONAL}

La percepción de beneficio funcional es muy importante para el turista. Se genera a través de la respuesta a una necesidad cogniti- va del individuo como consecuencia de su adaptación al entorno y puede ser definido como la utilidad adquirida a través del valor funcional (Babin, Darden, \& Griffin, 1994). El turista lo obtiene a través de la experiencia con los atributos del lugar (O’Leary \& Deegan, 2005). La persona impulsada por la búsqueda de soluciones, tiende a elegir la alternativa que tenga los mejores atributos según sus necesidades (Babin, Darden, \& Griffin, 1994). El beneficio funcional será mejor, cuanto más práctico sean los atributos del destino ante las necesidades de los turistas. Tendrán un mayor impacto en el turista, cuanto mejor sea la capacidad de respuesta ante las múltiples necesidades expresadas en su estadía (Sirgy \& $\mathrm{Su}, 2000$ ). Los atributos funcionales del destino turístico, lo harán más atractivo para ser visitado (Hosany \& Gilbert, 2010).

\section{PERCEPCIÓN DE BENEFICIO HEDÓNICO}

La percepción de beneficio hedónico es relevante para el turista. A través del mismo, evaluará el grado de placer obtenido en el lugar. Este surge como resultado de las experiencias sicosensoriales, desde la necesidad de estimulación y de búsqueda de sensaciones (Hirschman \& Holbrook, 1982). Es el placer obtenido a través de una experiencia con el servicio, asociada a las fantasías y sentimientos de las personas (Holbrook \& Hirschman, 1982). Representa experiencias multisensoriales, que son activadas a través de la estimulación de los sentidos. Los destinos turísticos son lugares formados por una mezcla de productos y servicios turísticos (Buhalis, 2000), que estimulan al visitante, al ofrecer experiencias sensoriales placenteras. Cuanto mejor sean las experiencias multisensoriales del turista, mayor será el placer experimentado en su estadía (Snepenger, Snepenger, Dalbey, \& Wessol, 2007). Como los destinos turísticos son esencialmente estéticos será muy importante el contacto visual del turista con el paisaje del lugar.

\section{PERCEPCIÓN DE BENEFICIO SIMBÓLICO}

El beneficio simbólico, es una característica del turista, que le permitirá apreciar el sentido de pertenencia que tiene el lugar visitado. Es aquel recibido por los múltiples componentes del autoconcepto, que es en gran medida el resultado de las evaluaciones de los demás, ya sean reales o irreales (Solomon, 1983) y está compuesto por una diversidad de representaciones que tiene cada persona y que están unidas a un conjunto particular de circunstancias sociales (Sirgy \& Su, 2000). El auto concepto está compuesto por cuatro aspectos: el real, el ideal, el social y el social ideal (Sirgy, 1982). El real es la manera en que el individuo se ve a sí mismo, el ideal es el modo como le gustaría ser, el social 
es la forma como el individuo cree que es visto por otras personas importantes, y el social ideal, es la manera en que a al individuo le gustaría ser visto por otras personas importantes (Sirgy \& Samli, 1985). El turista podría orientar su comportamiento en el lugar, ya sea para reforzar o mejorar su autoconcepto. Podría acudir a aquellos lugares que comuniquen un significado simbólico para sí mismo y para otros (Hosany \& Gilbert, 2010).

\section{SATISFACCIÓN CON EL DESTINO TURÍSTICO}

La satisfacción con el destino turístico es esencialmente lo que espera obtener el turista. Es el cumplimiento de la respuesta esperada (Oliver, 1999), que puede ser expresada a través de un estado afectivo (Baker \& Crompton, 2000) o también desde un estado cognitivo (De Rojas \& Camarero, 2008) o como una combinación de ambos (Del Bosque \& San Martin, 2008), posterior a las experiencias que el turista haya vivido en el lugar. Es una valoración positiva que el turista realiza de los beneficios obtenidos en el lugar (Yoon \& Uysal, 2005). Puede ser la suma de juicios de la calidad percibida por el turista (Stedman, 2002). La satisfacción es una opinión subjetiva que se sustenta en la evaluación realizada por el turista, después de haber vivido distintas experiencias en el lugar (Pizam \& Ellis, 1999).

\section{FAMILIARIDAD CON EL DESTINO TURÍSTICO}

La familiaridad del destino turístico es muy importante para el turista. Esta puede ser entendida como conocimiento, a menudo basado en interacciones y experiencias previas (Gefen, 2000). La familiaridad puede ser considerada como el conocimiento que la gente adquiere, basado en sus experiencias y contactos anteriores. Este conocimiento consigue reducir la incertidumbre y simplifica las relaciones con otras personas (Alba \& Hutchinson, 1987). La familiaridad cumple un rol muy importante en el proceso de elección (Gefen \& Straub, 2004) de los destinos turísticos. En la medida que el turista logra conocer mejor un lugar, le parecerá más familiar. La familiaridad se relaciona con los recuerdos agradables del turista, asociados al conocimiento de los atributos y de las imágenes del medio ambiente de los lugares que ha visitado (Hammitt, Kyle, \& Oh, 2009).

\section{HIPOTESIS}

La relación entre beneficios percibidos por el turista-satisfacción-familiaridad del lugar, ha sido escasamente analizada en la literatura de turismo. Como una contribución a la discusión del tema, este estudio plantea las siguientes hipótesis de estudio:

\section{Beneficio Funcional - Satisfacción}

Los atributos convertidos en beneficios funcionales proporcionan un mayor grado de satisfacción al consumidor (Veryzer Jr, 1995; Botti \& McGill, 2011) y pueden ser parte del diseño o de la estética del producto. El beneficio funcional adquirido por el turista a través de los atributos del lugar, podrían mejorar el estado de satisfacción del turista (Lee, Lee, \& Choi, 2011). Dada esta argumentación, es posible plantear la siguiente hipótesis:

H1: Cuanto mejor sea el beneficio funcional percibido por el turista, mayor será su grado de satisfacción en el destino turístico.

\section{Beneficio Hedónico - Satisfacción}

Las experiencias hedónicas pueden aumentar el grado de satisfacción del consumidor (Botti \& McGill, 2011). El consumidor hedónico tiene la tendencia a buscar experiencias placenteras que le brinden mayor satisfacción (Mano \& Oliver, 1993). El placer asociado a las experiencias de ocio influye en el estado de satisfacción del turista (De Rojas \& Camarero, 2008). El placer obtenido a través de los atractivos visuales del lugar podría incidir en el nivel de satisfacción del turista. Dada esta argumentación, es posible plantear la siguiente hipótesis:

H2: Cuanto mejor sea el beneficio hedónico percibido por el turista, mayor será su grado de satisfacción en el destino turístico.

\section{Beneficio Simbólico - Satisfacción}

El beneficio simbólico logrado por el turista se relaciona en forma directa y positiva con su satisfacción (Nam, Ekinci, \& Whyatt, 2011). Del mismo modo, incrementa su grado de satisfacción afectando con ello su comportamiento (Rogers, Peyton, \& Berl, 1992) en el lugar. El beneficio simbólico del turista podría mejorar el estado de satisfacción que le brinde el destino (Chon \& Olsen, 1991). El vínculo creado entre el beneficio simbólico y la satisfacción puede ayudar a percibir de mejor manera los servicios de hospitalidad (Ekinci, Dawes, \& Massey, 2008). Dada esta argumentación, es posible plantear la siguiente hipótesis:

H3: Cuanto mejor sea el beneficio simbólico percibido por el turista, mayor será su grado de satisfacción en el destino turístico.

\section{Satisfacción - Familiaridad}

La familiaridad es la expresión utilizada por las personas como resultado de su satisfacción (Van de Walle, Turner, \& Davenport, 
2003) después de vivir una experiencia. La satisfacción influye en la familiaridad (Casaló, Flavián, \& Guinalíu, 2008). La satisfacción sustenta el vínculo entre el lugar y el turista e incide para que este lugar le sea más familiar (Horng, Liu, Chou, \& Tsai, 2012), más aún si es lejano (Prentice, 2004), determinando la intención y actitud de viajar a él. Dada esta argumentación, es posible plantear la siguiente hipótesis:

H4. Cuanto mayor sea el grado de satisfacción del turista, más familiar le será el destino turístico. En forma esquemática las hipótesis de este estudio son:

Figura 1. Hipótesis

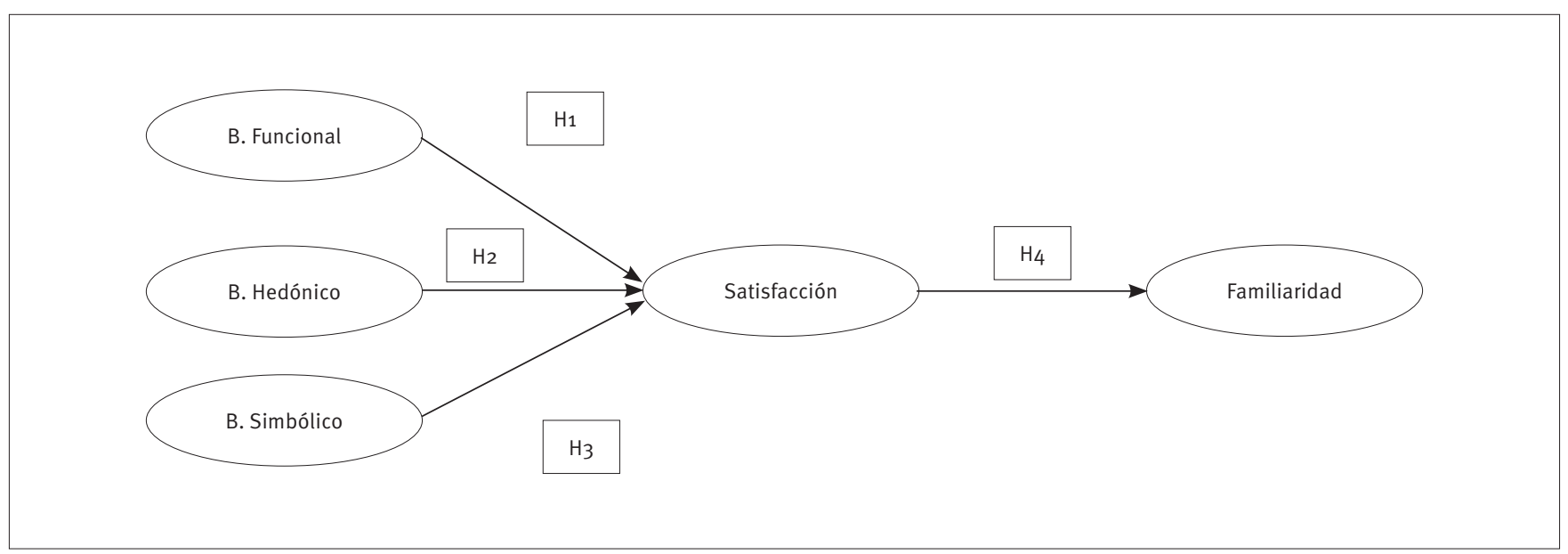

NOTA: B: Beneficio

\section{METODOLOGIA}

Con el objetivo de identificar la escala más idónea para medir las relaciones en la que la satisfacción es una dimensión determinante de la familiaridad y que a su vez es una consecuencia de la percepción de beneficios funcionales, hedónicos y simbólicos que el turista perciba en el destino turístico, con un buen grado de fiabilidad, validez y dimensionalidad, se desarrolló un proceso en diferentes etapas (Deng \& Dart, 1994). La primera etapa consistió en construir escalas con un grado de validez de contenido. Para ello se realizó un exhaustivo análisis de la literatura considerando las escalas construidas en diversos estudios previos, por ejemplo, Kennedy, Ferrell y Leclair, (2001); Gefen (2000); Coulter y Coulter (2003); Van der Heijden y Verhagen (2003); Ha y Perks (2005); Walmsley y Young (1998); Babin y Darden (1995); Park (2004); Chitturi, Raghunathan y Mahajan, (2008); Kim, Forsythe, Gu y Moon, (2002); Babin, Chebat, y Michon, (2004); Babin, Lee, Kim y Griffin, (2005); Dhar y Wertenbroch (2000); Mathwick, Malhotra y Rigdon, (2001); Brockman (1998).

Posteriormente se realizó un estudio de incidentes críticos, donde las personas debían describir aquellos factores que formaban parte de los constructos analizados. En el estudio participaron 40 personas elegidas en una muestra no probabilística por conveniencia y se obtuvieron las escalas previas de fami- liaridad, satisfacción, beneficio simbólico, beneficio funcional y beneficio hedónico. A continuación, se realizó un segundo proceso de depuración de estas escalas recomendado por De Wulf y Odekerken-Schörder, (2003). Se realizaron una serie de focus groups compuestos por turistas habituales de diferentes sectores de Chile y, además, diferentes entrevistas con expertos en turismo y ejecutivos (as) comerciales de agencias de turismo. Estos análisis permitieron sumar aquellos indicadores que reflejan más adecuadamente cada una de las dimensiones dentro del contexto de estudio, y readecuar y/o eliminar aquellos indicadores que resultaron ser conflictivos o redundantes. Para ello, se utilizó una modificación del método de Zaichkowsky, (1985). Cada experto debió calificar cada ítem respecto a su dimensión, considerando tres alternativas: claramente, algo o nada representativo. Finalmente, se decidió conservar aquellos ítems en los que existía un alto nivel de consenso (Lichtenstein, Netemeyer, \& Burton, 1990). Con estos análisis se obtuvieron las escalas con las cuales se construyó el cuestionario.

En la segunda etapa, se construyó el cuestionario definitivo. Con este cuestionario preliminar se le realizó un pre test cuantitativo a una muestra aleatoria de 40 personas y, posteriormente, con esos datos se realizó un análisis factorial exploratorio y se calculó el Alpha de Cronbach a cada una de las dimensiones resultantes. Con este análisis previo se pudo confirmar la existencia de cada una de las dimensiones que resultaron de los aná- 
lisis precedentes. Los ítems se redactaron como afirmaciones y debían ser respondidos por medio de una escala Likert de 7 puntos (ver tabla 1). Todas fueron redactadas de tal forma que pudieran ser entendidas y respondidas por todos los entrevistados.

\section{TABLA 1. Escalas de Medida}

\begin{tabular}{l|l}
\hline \multicolumn{2}{l}{ BENEFICIO FUNCIONAL (B. FUNCIONAL) } \\
\hline B. Funcional 1 & $\begin{array}{l}\text { En este lugar logré vacacionar justo como lo } \\
\text { necesitaba }\end{array}$ \\
\hline B. Funcional 2 & $\begin{array}{l}\text { En este lugar encontré las vacaciones que estaba } \\
\text { buscando }\end{array}$ \\
\hline B. Funcional 3 & $\begin{array}{l}\text { Siempre es conveniente venir de vacaciones a } \\
\text { este lugar }\end{array}$ \\
\hline B. Funcional 4 & $\begin{array}{l}\text { Comparado con otros lugares similares, este es el } \\
\text { mejor lugar para vacacionar }\end{array}$ \\
\hline
\end{tabular}

\section{BENEFICIO SIMBÓLICO (B. SIMBÓLICO)}

\begin{tabular}{l|l}
\hline B. Simbólico 1 & Este lugar refleja lo que soy \\
\hline B. Simbólico 2 & Este lugar es coherente con cómo me veo a mi mismo \\
\hline B. Simbólico 3 & $\begin{array}{l}\text { Me identifico con las personas que prefieren este } \\
\text { lugar }\end{array}$ \\
\hline B. Simbólico 4 & A este lugar viene gente similar a mí \\
\hline
\end{tabular}

BENEFICIO HEDÓNICO ATRACTIVOS VISUALES (B. HEDAV.)

\begin{tabular}{l|l}
\hline B. Hedav. 1 & Este lugar me encanta por sus formas \\
\hline B. Hedav. 2 & Este lugar me encanta por su estética \\
\hline B. Hedav. 3 & Este lugar es un placer para mis sentidos \\
\hline B. Hedav. 4 & Me encanta observar todos sus sitios \\
\hline FAMILIARIDAD (FAM.) \\
\hline
\end{tabular}

\begin{tabular}{l|l}
\hline Fam. 1 & Este lugar me es muy familiar \\
\hline Fam. 2 & Este lugar me es muy conocido \\
\hline Fam. 3 & $\begin{array}{l}\text { Sobre este lugar siempre estoy muy bien } \\
\text { informado }\end{array}$ \\
\hline Fam. 4 & Sobre este lugar siempre estoy consiente \\
\hline Fam. 5 & $\begin{array}{l}\text { Mis amigos y familiares dicen que conozco muy } \\
\text { bien este lugar }\end{array}$ \\
\hline SATISFACCIÓN (SAT.) \\
\hline Sat.1 & Este lugar es el mejor que yo he visitado \\
\hline Sat. 2 & Este lugar es lo que yo esperaba \\
\hline Sat. 3 & Este lugar colma mis expectativas \\
\hline Sat. 4 & Este lugar es tal como lo había pensado \\
\hline Sat. 5 & Este lugar ha sido mi mejor elección \\
\hline
\end{tabular}

Se utilizó un muestreo no probabilístico con base en cuotas, en proporción a los destinos turísticos visitados en Chile según el Servicio Nacional de Turismo (SERNATUR), divididos en playas (36\%), lagos (25\%) y otros atractivos turísticos (39\%). Las encuestas fueron aplicadas durante los meses de mayo y junio de 2009 en ciudades de Chile, tales como Santiago, Arica, Iquique, La Serena, Viña del Mar, Curicó Concepción y Temuco.

En la tercera etapa, se recolectaron los datos. La encuesta fue aplicada a una muestra total de 750 personas, considerando como referencia para responder el último lugar turístico en que pernoctaron.

\section{Análisis Sicométrico de los datos}

Con los datos obtenidos se realizó un análisis sicométrico, hasta obtener escalas con un buen grado de fiabilidad, validez y dimensionalidad. Se aplicó un análisis factorial exploratorio, un análisis factorial confirmatorio (SEM), y diversos análisis de fiabilidad con Alpha de Cronbach, Fiabilidad del Costructo y Varianza Extraída (AVE).

Para identificar aquellos ítems que no están adheridos a su dimensión, se realizaron análisis factoriales de componentes principales con rotación varimax (Hair, Anderson, Tatham, \& Black, 1998).

Según este procedimiento, no fue necesario eliminar indicadores de las escalas analizadas (ver tabla 2). De hecho, todas presentaron un buen grado de unidimensionalidad, con cargas factoriales que superan ampliamente el 0,4 (Larwood, Falbe, Kriger, \& Miesing, 1995).

Considerando las diferentes escalas, a través de ecuaciones estructurales, se desarrolló un Análisis Factorial Confirmatorio para corroborar si los indicadores o variables eran adecuados para lograr un buen ajuste del modelo. Los requisitos considerados fueron los tres criterios propuestos por Jöreskog y Sörbom (1993).

El primer criterio consiste en eliminar aquellos indicadores que tengan una débil condición de convergencia con su correspondiente variable latente. Se utilizó como exigencia una $t$ de student mayor a 2,28 ( $p=0,01)$. El segundo criterio consiste en apartar del análisis aquellas variables cuyas cargas traducidas en coeficientes estandarizados son menores a 0,5. Por último, se deben eliminar aquellos indicadores que presenten una relación lineal R2 menor a o,3. En este proceso se utilizó el paquete estadístico AMOS SPSS en su versión 18. Para este análisis, no se eliminaron indicadores de acuerdo a ninguno de los tres criterios. Los índices de ajuste de este modelo Factorial Confirmatorio fueron aceptables: IFI 0,916, CFI 0,916, RMSEA 0,084, Normed X2 6,33. 
TABLA 2. Análisis Factorial Exploratorio de las Escalas

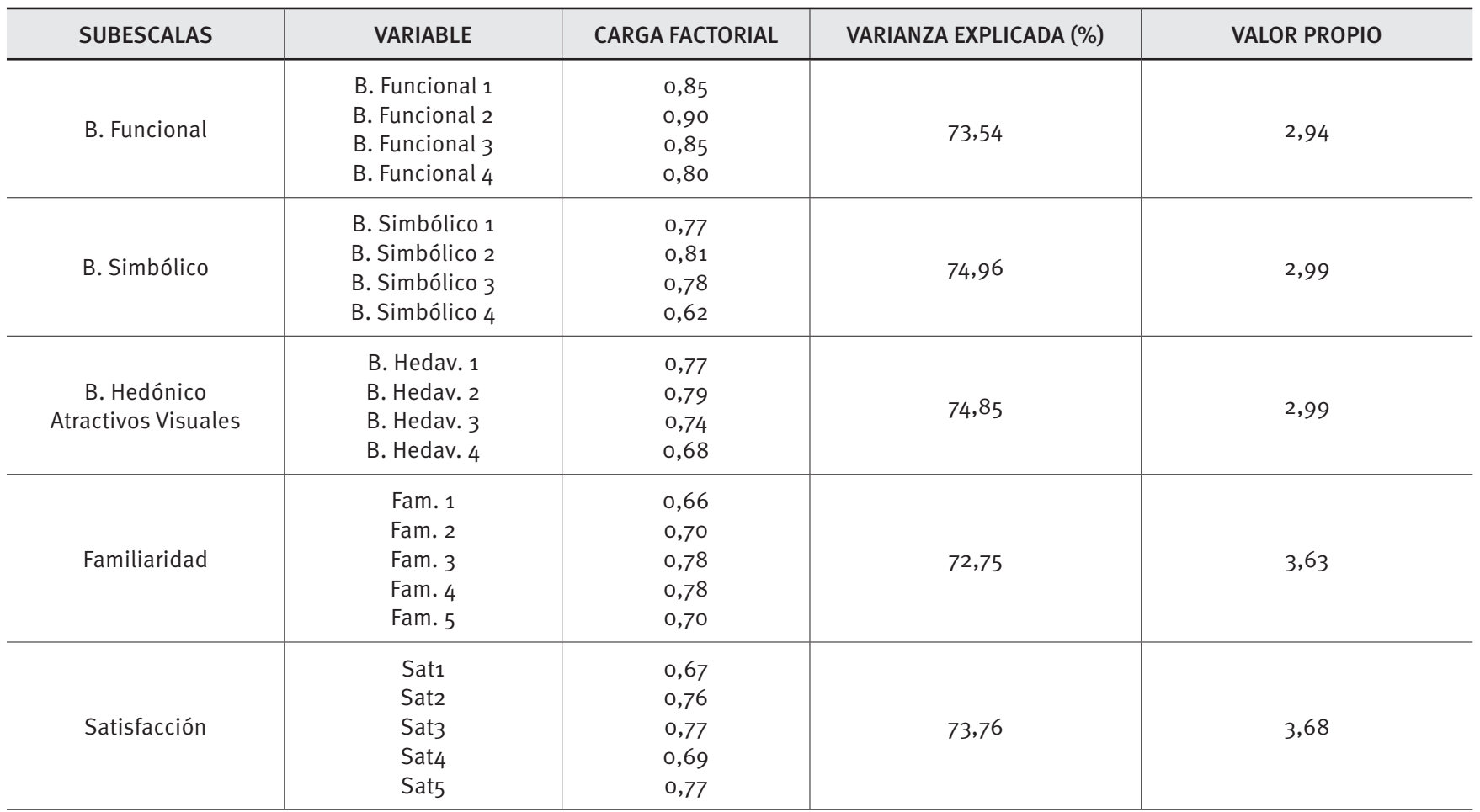

Una vez verificado el modelo óptimo, se comprobó la fiabilidad de cada una de las escalas. Para ello se aplicaron tres test: Alpha de Cronbach (límite 0,7), Fiabilidad Compuesta del Constructo (límite 0,7) (Jöreskog, 1971) y Análisis de la Varianza Extraída (límite 0,5) (Fornell \& Larcker, 1981). Los resultados muestran que en todos los casos se cumplen los valores mínimos establecidos (ver tabla 3) por estos parámetros de fiabilidad.

\section{TABLA 3. Fiabilidad de las Escalas}

\begin{tabular}{|c|c|c|c|c|}
\hline SUBESCALAS & VARIABLE & $\begin{array}{l}\text { ALPHA DE } \\
\text { CRONBACH }\end{array}$ & $\begin{array}{l}\text { FIABILIDAD DE } \\
\text { CONSTRUCTO }\end{array}$ & VARIANZA EXTRAÍDA \\
\hline B. Funcional & $\begin{array}{l}\text { B. Funcional } 1 \\
\text { B. Funcional } 2 \\
\text { B. Funcional } 3 \\
\text { B. Funcional } 4\end{array}$ & 0,87 & 0,88 & 0,65 \\
\hline B. Simbólico & $\begin{array}{l}\text { B. Simbólico } 1 \\
\text { B. Simbólico } 2 \\
\text { B. Simbólico } 3 \\
\text { B. Simbólico } 4\end{array}$ & 0,88 & 0,89 & 0,66 \\
\hline $\begin{array}{c}\text { B. Hedónico Atractivos } \\
\text { Visuales }\end{array}$ & $\begin{array}{l}\text { B. Hedav. } 1 \\
\text { B. Hedav. } 2 \\
\text { B. Hedav. } 3 \\
\text { B. Hedav. } 4\end{array}$ & 0,88 & 0,89 & 0,67 \\
\hline Satisfacción & $\begin{array}{l}\text { Sat1 } \\
\text { Sat2 } \\
\text { Sat3 } \\
\text { Sat4 } \\
\text { Sat5 }\end{array}$ & 0,90 & 0,91 & 0,67 \\
\hline
\end{tabular}


La validez se comprobó, considerando la validez de contenido y de constructo. Las escalas presentan un buen grado de validez de contenido, debido a que se realizó un profundo análisis de la literatura, un estudio de incidentes críticos con turistas, y posteriormente, una depuración de esta escala a través de diferentes grupos focales con turistas y entrevistas en profundidad realizadas a expertos (as) y ejecutivos (as) comerciales de Agencias de Turismo. Para cumplir con la validez de constructo se analizó si la escala propuesta ya depurada, cumple con la validez convergente y discriminante.

La validez convergente se confirmó al observar que todos los coeficientes estandarizados del análisis factorial confirmatorio (AFC), resultaron estadísticamente significativos al 0,01 y mayores a 0,5 (Bagozzi \& Yi, 1988). Para comprobar la existencia de validez discriminante, se utilizó el test de intervalos de confianza (Anderson \& Gerbin, 1988). Este consiste en construir los intervalos de confianza resultantes de las correlaciones entre las diferentes variables latentes que componen el modelo factorial confirmatorio (AFC) (ver tabla 4). De acuerdo a este test, el modelo presenta validez discriminante, ya que en ningún intervalo de confianza se contuvo el valor 1 (Bagozzi, 1981).
De hecho, en todos los casos, las correlaciones se alejan considerablemente de ese valor.

\section{TABLA 4. Test de Intervalos de Confianza}

\begin{tabular}{c|c}
\hline RELACIÓN BIVARIADA & INTERVALOS DE CONFIANZA \\
\hline Funcional - Simbólico & $0,61-0,62$ \\
\hline Satisfacción - Hedónico & $0,68-0,69$ \\
\hline Funcional - Hedónico & $0,68-0,69$ \\
\hline Satisfacción - Simbólico & $0,61-0,62$ \\
\hline Familiaridad - Funcional & $0,55-0,55$ \\
\hline Familiaridad - Simbólico & $0,55-0,56$ \\
\hline Familiaridad - Satisfacción & $0,51-0,52$ \\
\hline Satisfacción - Funcional & $0,83-0,84$ \\
\hline Hedónico - Simbólico & $0,60-0,61$ \\
\hline
\end{tabular}

Con todos estos antecedentes podemos concluir que el modelo propuesto presenta un buen grado de validez general.

\section{RESULTADOS}

Las cuatro hipótesis fueron testeadas utilizando un modelo de ecuaciones estructurales (SEM) (Bagozzi, 1981). Como se puede observar, a través de los $\beta$ estandarizados, en la Figura 2, la satisfacción tiene un efecto directo y positivo ( $\beta$ o,55; p<o,001) sobre la familiaridad $(\mathrm{R} 20,30)$. Esto es muy relevante para el turismo dado que, la satisfacción del turista es la base para que el lugar le sea más familiar, más aún si es lejano. El beneficio funcional ( $\beta$ o,63; p<0,001), el beneficio hedónico ( $\beta$ o,18; $p<0,001)$, y el beneficio simbólico $(\beta$ o,13; p<o,001) tienen un efecto directo y positivo sobre la satisfacción $(\mathrm{R} 2$ o,74). No obstante, es necesario observar con cautela la superposición del beneficio funcional por sobre el beneficio hedónico y el beneficio simbólico.

\section{Figura 2. Resultado Relaciones Causales}

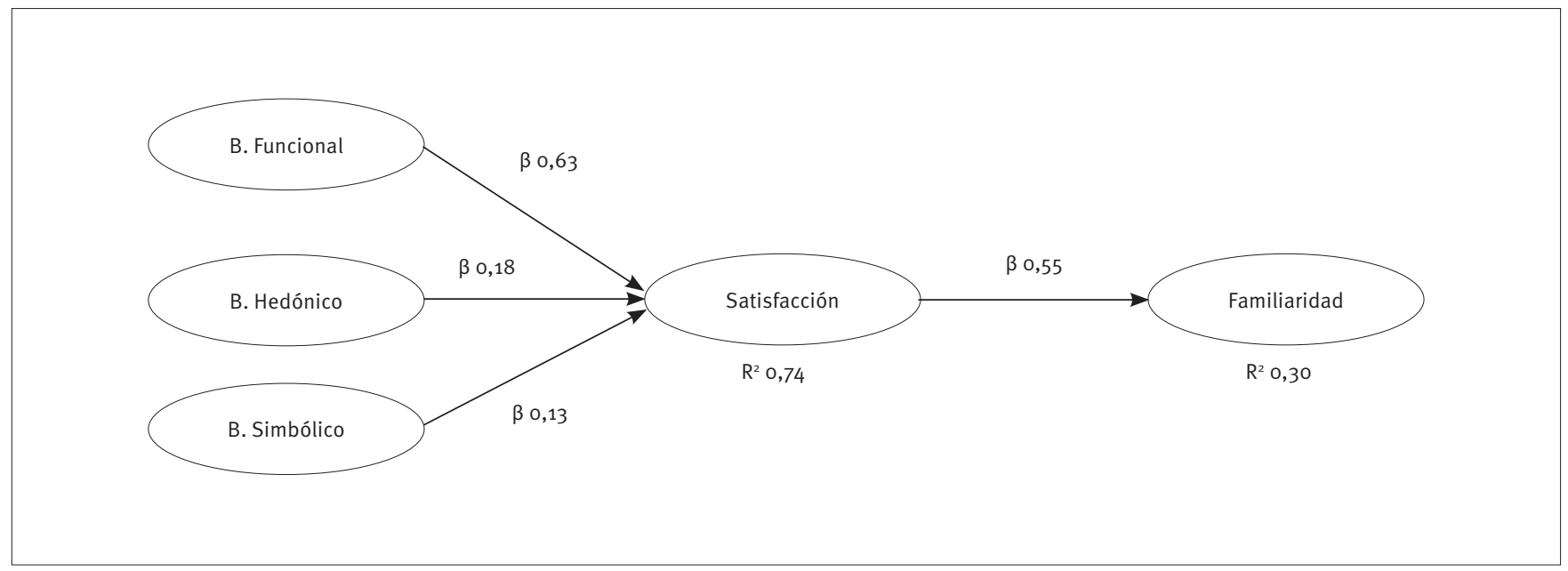

NOTA: B: Beneficio 
Como ha sido demostrado, el $55 \%$ de la familiaridad de un destino turístico es explicada por la satisfacción del turista. La satisfacción se explica por un $65 \%$ de beneficio funcional, un 18\% de beneficio hedónico y un $13 \%$ de beneficio simbólico. Se observaron correlaciones positivas y significativas entre los constructos. (ver tabla 5).

\section{TABLA 5. Matriz de Correlaciones}

\begin{tabular}{|c|c|c|c|c|c|}
\hline B. Funcional & 1 & 0,620 & 0,577 & 0,764 & 0,532 \\
\hline B. Hedónico & & 1 & 0,552 & 0,625 & 0,470 \\
\hline Satisfacción & & & & 1 & 0,479 \\
\hline Familiaridad & & & & & 1 \\
\hline
\end{tabular}

Nota: La Correlación es significativa al 0,01\%.

A partir de estos antecedentes, la satisfacción puede cumplir un rol mediador entre la percepción de beneficios y la familiaridad. El efecto mediador se produce cuando una tercera variable, variable mediadora (VMe), es capaz de influir en la relación entre una variable independiente (VI) y otra variable dependiente (VD) (Baron \& Kenny, 1986). Para comprobar si la satisfacción asume un rol mediador, se plantearon tres estructuras de mediación: 1) beneficio funcional - satisfacción - familiaridad; 2) beneficio hedónico - satisfacción - familiaridad; 3) beneficio simbólico - satisfacción - familiaridad.

Estas estructuras fueron evaluadas en base a algunas condiciones de mediación (ver tabla 6), por ejemplo, un análisis de dos caminos de mediación (Baron \& Kenny, 1986; Vieira, 2008). Para este análisis, se debe comprobar si al evaluar en forma aislada: 1) la VI afecta significativamente a la VMe; 2) la VI afecta significativamente a la VD, sin la presencia de la VMe; 3) la VMe tiene un efecto único significativo sobre la VD; o si al evaluar en forma conjunta: 4) el efecto de la VI sobre la VD disminuye después de incorporar la VMe. Si los valores $\beta$ obtenidos como resultado de las relaciones entre (VI-VMe); (VI-VD) y (VMe-VD) (tres primeros pasos), y si el valor $\beta$ obtenido como resultado de la relación entre (VI-VD), disminuye con la presencia VMe (cuarto paso), existe evidencia del rol mediador de la satisfacción. Del mismo modo, fue necesario aplicar el test de dos caminos de mediación Sobel, (1982); Aroian, (1944/1947) y Goodman (1960) que permite comprobar la fuerza de la mediación entre la VI y la VD (Anabila, Narteh, \& Koduah, 2012),

Se obtuvieron valores $\beta$ significativos ( $p<0,000)$, para todas las estructuras de mediación (tres primeros pasos). Se pudo identificar una reducción (ver $\beta$ ) del impacto de la variable independiente sobre la variable dependiente en presencia de la variable mediadora (cuarto paso). Es así que, cuando en la relación beneficio funcional-satisfacción-familiaridad, no existe la presencia de la satisfacción, el efecto del beneficio funcional sobre la familiaridad es de $(\beta 0,53 ;$ p $<0,000)$. Distinto es al incorporar la satisfacción como mediadora, el impacto del beneficio funcional sobre la familiaridad disminuye a $(\beta \circ, 38 ; p<0,000 ; \Delta 0,15)$. La misma situación se repite en la relación entre beneficio hedónico-satisfacción-familiaridad, sin satisfacción ( $\beta 0,50$; p<0,000); con satisfacción disminuye a $(\beta 0,29 ; p<0,000 ; \Delta 0,21)$ y en la relación entre el beneficio simbólico-satisfacción-familiaridad, sin satisfacción ( $\beta$ 0,54; $p<0,000)$; con satisfacción disminuye a $(\beta 0,37$; p $<0,000 ; \Delta 0,17)$. Esto indica que la satisfacción absorbe gran parte del impacto de los beneficios sobre la familiaridad, dejando en evidencia el importante rol mediador que cumple en estas relaciones.

Las pruebas estadísticas de mediación de Sobel, (1982); Aroian, (1944/1947) y Goodman, (1960) con sus respectivos valores $Z \neq 0$ y valores $p$ asociados indican una fuerte evidencia de la mediación de la satisfacción en las relaciones entre los beneficios y la familiaridad.

De acuerdo al modelo teórico propuesto fueron realizados los cálculos de los efectos indirectos. Los efectos indirectos se producen al existir una tercera variable en el medio de esa relación. Los valores $\beta$ estandarizados obtenidos (ver tabla 7), sugieren un impacto indirecto y significativo de los beneficios sobre la familiaridad. 
TABLA 6. Hipótesis de Mediación del Modelo Propuesto

\begin{tabular}{|c|c|c|c|c|c|c|c|c|c|c|c|c|c|}
\hline \multicolumn{8}{|c|}{ ANÁLISIS: CAMINOS DE MEDIACIÓN } & \multicolumn{6}{|c|}{ TESTS DE SOBEL, AROIAN Y GOODMAN } \\
\hline \multirow[b]{2}{*}{ CONDICIÓN } & \multirow{2}{*}{$\begin{array}{l}\text { V. INDEPEN- } \\
\text { DIENTE } \\
(\mathrm{VI})\end{array}$} & \multirow{2}{*}{$\begin{array}{l}\text { V. DEPEN- } \\
\text { DIENTE } \\
\text { (VD) }\end{array}$} & \multirow[b]{2}{*}{$\begin{array}{l}\beta \text { ESTAN- } \\
\text { DARIZADO }\end{array}$} & \multirow{2}{*}{$\begin{array}{c}\beta \text { NO } \\
\text { ESTANDA- } \\
\text { RIZADO }\end{array}$} & \multirow{2}{*}{$\begin{array}{c}\text { ERROR } \\
\text { ESTAN-DAR }\end{array}$} & \multirow[b]{2}{*}{ VALOR $p$} & \multirow[b]{2}{*}{$\mathrm{R}^{2} \%$} & \multicolumn{2}{|c|}{ SOBEL } & \multicolumn{2}{|c|}{ AROIAN } & \multicolumn{2}{|c|}{ GOODMAN } \\
\hline & & & & & & & & $\begin{array}{c}\text { VALOR } \\
\text { Z }\end{array}$ & $\begin{array}{c}\text { VALOR } \\
p\end{array}$ & $\begin{array}{c}\text { VALOR } \\
\text { Z }\end{array}$ & $\begin{array}{c}\text { VALOR } \\
\mathrm{p}\end{array}$ & $\begin{array}{c}\text { VALOR } \\
\mathrm{Z}\end{array}$ & $\begin{array}{c}\text { VALOR } \\
p\end{array}$ \\
\hline \multirow{3}{*}{ Aislada } & B. Funcional & Satisfacción (VMe) & 0,82 & 0,99 & 0,05 & 0,000 & 68 & \multirow{5}{*}{1,90} & \multirow{5}{*}{0,085} & \multirow{5}{*}{1,89} & \multirow{5}{*}{0,086} & \multirow{5}{*}{1,92} & \multirow{5}{*}{0,054} \\
\hline & B. Funcional & Familiaridad & 0,53 & 0,55 & 0,04 & 0,000 & 28 & & & & & & \\
\hline & $\begin{array}{c}\text { Satisfacción } \\
\text { (VMe) }\end{array}$ & Familiaridad & 0,51 & 0,42 & 0,03 & 0,000 & 26 & & & & & & \\
\hline \multirow[b]{2}{*}{ Conjunta } & B. Funcional & Familiaridad & 0,38 & 0,37 & 0,07 & 0,000 & \multirow[b]{2}{*}{31} & & & & & & \\
\hline & $\begin{array}{c}\text { Satisfacción } \\
\text { (VMe) }\end{array}$ & Familiaridad & 0,19 & 0,15 & 0,06 & 0,010 & & & & & & & \\
\hline \multirow{3}{*}{ Aislada } & B. Hedónico & Satisfacción (VMe) & 0,68 & 1,05 & 0,06 & 0,000 & 46 & \multirow{5}{*}{2,85} & \multirow{5}{*}{0,004} & \multirow{5}{*}{2,82} & \multirow{5}{*}{0,004} & \multirow{5}{*}{2,87} & \multirow{5}{*}{0,003} \\
\hline & B. Hedónico & Familiaridad & 0,50 & 0,66 & 0,05 & 0,000 & 25 & & & & & & \\
\hline & $\begin{array}{c}\text { Satisfacción } \\
\text { (VMe) }\end{array}$ & Familiaridad & 0,51 & 0,42 & 0,03 & 0,000 & 26 & & & & & & \\
\hline \multirow[b]{2}{*}{ Conjunta } & B. Hedónico & Familiaridad & 0,29 & 0,38 & 0,06 & 0,000 & \multirow[b]{2}{*}{31} & & & & & & \\
\hline & $\begin{array}{c}\text { Satisfacción } \\
\text { (VMe) }\end{array}$ & Familiaridad & 0,31 & 0,25 & 0,04 & 0,000 & & & & & & & \\
\hline \multirow{3}{*}{ Aislada } & B. Simbólico & Satisfacción (VMe) & 0,61 & 0,79 & 0,06 & 0,000 & 37 & \multirow{5}{*}{2,57} & & & & & \\
\hline & B. Simbólico & Familiaridad & 0,54 & 0,58 & 0,04 & 0,000 & 30 & & & & & & \\
\hline & $\begin{array}{c}\text { Satisfacción } \\
\text { (VMe) }\end{array}$ & Familiaridad & 0,51 & 0,42 & 0,03 & 0,000 & 26 & & & & & 26 & \\
\hline & B. Simbólico & Familiaridad & 0,37 & 0,40 & 0,05 & 0,000 & & & & & & & \\
\hline Conjunta & $\begin{array}{c}\text { Satisfacción } \\
\text { (VMe) }\end{array}$ & Familiaridad & 0,28 & 0,23 & 0,03 & 0,000 & $35 \%$ & & & & & & \\
\hline
\end{tabular}

Nota: (VMe): Variable Mediadora

\section{TABLA 7. Efectos Indirectos}

\begin{tabular}{ll|c|c}
\hline \multicolumn{2}{l|}{ RELACIONES INDIRECTAS } & $\beta$ ESTANDARIZADO & VALOR $p$ \\
\hline B. Funcional & Familiaridad & 0,35 & $0,000^{*}$ \\
\hline B. Hedónico & Familiaridad & 0,10 & $0,000^{*}$ \\
\hline B. Simbólico & Familiaridad & 0,07 & $0,000^{*}$ \\
\hline
\end{tabular}

*Nota: p<o,05

La Satisfacción está en medio de la relación indirecta entre beneficio funcional-familiaridad; beneficio hedónico-familiaridad; beneficio simbólico-familiaridad. Para este caso, no siempre es así, coincide que el efecto mediador y el efecto indirecto se da entre una VD y una VI.

\section{CONCLUSIONES}

En este estudio fue posible comprobar que, para el turista, es muy importante asociar la familiaridad del destino turístico con su satisfacción. La satisfacción cumple un importante rol mediador entre la percepción de beneficios del turista y la familiaridad del lugar. El turista podrá tener la mejor percepción de beneficios funcionales, hedónicos y simbólicos, sin embargo, de nada servirá si no se siente satisfecho con su estadía en el lugar. Para que un destino sea percibido como un lugar familiar, es necesario que el turista viva experiencias que fomenten su satisfacción. El estado de satisfacción ayudará al turista a relacionar de mejor manera la percepción de beneficios funcionales, hedónico, simbólicos con la familiaridad. Para un turista estar familiarizado con 
el lugar, le será de gran ayuda en su proceso de elección, dado que influirá en la imagen que de él se forme, en el apego que sienta hacia él, por lo que se transformará en un importante antecedente del placer que experimente en su potencial estadía. Los principales grupos de interés de la industria turística, deberían pre-ocuparse de garantizar la satisfacción del turista como un medio para lograr su familiarización con el lugar.

No obstante, es imprescindible que el destino turístico sea percibido como un lugar que otorga un alto nivel de beneficios funcionales, dado que esto tiene mayor importancia para el turista frente a la percepción de beneficios hedónicos y simbólicos. El cuidado del medio ambiente, la comodidad de las instalaciones, así como la disponibilidad y facilidad para acceder a los servicios básicos, ayudarán a mejorar la percepción de beneficios funcionales. Esto servirá de base para decantar una buena percepción de beneficios hedónicos y simbólicos. Ejemplo de ello es la puesta en escena de los distintos resort de la "Riviera Maya” en México. No sólo sus aguas turquesas y finas arenas serán parte del beneficio funcional que espera obtener el turista, sino que además, le importará la higiene de sus alimentos e instalaciones, la disponibilidad y facilidad de cajeros automáticos y la disponibilidad de medios de transporte expeditos.

Del mismo modo, es importante que, el destino turístico sea percibido como un lugar que ofrece beneficios hedónicos. Para ello es necesario que los grupos de interés redoblen sus esfuerzos para cuidar los detalles estéticos del destino y se ocupen, tanto del diseño, como de las formas, para mantener un equilibrio entre lo natural y lo creado por el hombre. Desde el punto de vista estético, la omisión de no cuidar estos equilibrios puede ser nefasta. Ejemplo de ello es lo ocurrido en la ciudad de Valparaíso, Chile. Esta ciudad, en el año 2003, fue declarada patrimonio de la humanidad, principalmente por su belleza arquitectónica. Sin embargo, en los últimos años, se han levantado edificios en el borde costero, que provocan una asimetría estética a la vista del turista y de sus residentes, quienes perdieron la "vista al mar”. El equilibrio entre desarrollo urbano y desarrollo turístico, debería ser la base fundamental para mantener el placer de lo estético para el turista y para los residentes del lugar. Muy por el contrario, distinta es la situación que presenta Machu Pichu, en Perú. Hasta hoy se conservan y mantienen sus cualidades arquitectónicas y paisajísticas originales, lo que concede al turista el placer de observar los atractivos visuales del lugar.

Es necesario que el destino turístico sea percibido como un lugar que entrega beneficios simbólicos. Los grupos de interés, deberían conocer el perfil de sus visitantes, con el fin de poner a disposición de los mismos, productos y servicios que refuercen su “yo". El turista estará pendiente de lo que piensan los demás sobre él, tratará de buscar en el destino turístico las señales que le den respuesta a ello. Como por ejemplo Río de Janeiro, Brasil. Río, no es sólo playa y sol, sino que, además, se caracteriza a nivel internacional por sus atributos paisajísticos y culturales. La atracción natural “Pan de Azúcar”, el “Cristo Redentor”, el “Estadio Maracaná” o el "Parque Nacional de Tijuca” (declarado Reserva de la Biosfera), se convierten en alternativas válidas para el turista a la hora de fortalecer sus diferentes “yo”. En concreto, la relación entre beneficios y satisfacción puede ser muy importante para que el destino turístico logre generar lazos familiares con el turista.

En relación a las implicancias para la gestión, las autoridades deberían considerar la triada de beneficios funcionales, hedónicos y simbólicos en forma escalonada, pero, como un todo, a la hora de establecer estrategias de marketing turístico que busquen potenciar dicha satisfacción, con el fin de que el lugar sea percibido como más familiar que otros.

\section{LIMITACIONES}

Dada la especificidad de la muestra, (el segmento juvenil chileno), que si bien tiene su mérito, por causa del efecto cultural, puede producirse cierto sesgo, lo que dificulta la extrapolación de los resultados de este estudio, a otros destinos turísticos internacionales

Si bien la relación percepción de beneficios-satisfacciónfamiliaridad no ha sido muy estudiada en el sector turístico, se requiere mucha más rigurosidad metodológica para obtener resultados que permitan una interpretación más firme de los datos.

\section{FUTURAS LÍNEAS DE INVESTIGACIÓN}

Los autores sugieren estudiar el impacto de la satisfacción como variable mediadora, sobre otros factores de marketing, junto a la familiaridad, como por ejemplo, sobre la confianza del turista hacia el destino turístico.

Los autores sugieren profundizar el estudio de la relación entre percepción de beneficios - satisfacción - familiaridad, en el sector turístico, utilizando para ello, un muestreo probabilístico, como por ejemplo, aleatorio simple.

\section{NOTA DE AGRADECIMENTO}

Los autores agradecen la ayuda concedida por el Proyecto UAM-Santander 2013-2014 y también la ayuda proveniente del Ministerio de Economía y Competitividad de España (Ref. N.ECO2012-36032-Co3-01). 


\section{REFERENCIAS}

Alba, J. W \& Hutchinson, J. W. (1987). Dimensions of consumer expertise. Journal of Consumer Research, 13(4), 411-454

Anabila, P, Narteh, B, \& Koduah, E. Y. T. (2012). Relationship marketing practices and customer loyalty: evidence from the banking industry in Ghana. European Journal of Business and Management, 4(13), 51-62.

Anderson, F. C, \& Gerbing, D. W. (1988). Structural equation modeling in practice: a review and recommended two-step approach. Psychological Bulletin, 103(3), 411-423.

Andsager, J. L, \& Drzewiecka, J. A. (2002). Desirability of differences in destinations. Annals of Tourism Research, 29(2), 401-421.

Aroian, L. A. (1944/1947). The probability function of the product of two normally distributed variables. Annals of Mathematical Statistics, 18, 265-271.

Babin, B. J, Darden, W. R, \& Griffin, M. (1994). Work and/or fun: measuring hedonic and utilitarian shopping value. Journal of Consumer Research, 20(4), 644-656.

Babin, L, \& Darden, W. R. (1995). Consumer self-regulation in a retail environment. Journal of Retailing, 71(1), 47-70.

Babin, B. J, Chebat, J. C, \& Michon, R. (2004). Perceived appropriateness and its effect on quality, affect and behavior. Journal of Retailing and Consumer Services, 11(5), 287-298.

Babin, B. J, Lee, Y. K, Kim, E. J, \& Griffin, M. (2005). Modeling consumer satisfaction and word-of- mouth: restaurant patronage in Korea. The Journal of Services Marketing, 19(3), 133-139.

Bagozzi, R. P. (1981). Attitudes, intentions, and behavior: a test of some key hypotheses. Journal of Personality and Social Psychology, 41(4), 607-627.

Bagozzi, R. \& Yi, Y. (1988). On the evaluation of structural equation models. Journal of Marketing Science, 6(1), 74-94.

Baker, D. A \& Crompton, J. L. (2000). Quality, satisfaction and behavioral intentions. Annals of Tourism Research, 27(3), 785-804.

Baron, R. M \& Kenny, D. A. (1986). The moderator - mediator variable distinction in social psychological research: conceptual, strategic, and statistical considerations. Journal of Personality and Social Psychology, 51(6), 1173-1182.

Bigné, J. E, Sánchez, M. I, \& Sánchez, J. (2001). Tourism image, evaluation variables and after purchase behavior: inter-relationship. Tourism Management, 22(6), 607-616.

Botti, S \& McGill, A. L. (2011). The locus of choice: personal causality with hedonic and utilitarian decisions. Journal of Consumer Research, $37(6), 1065-1078$.

Brockman, B. (1998). The influence of affective state on satisfaction ratings. Journal of Consumer Satisfaction/Dissatisfaction and Complaining Behavior, 11, 40-50.

Buhalis, D. (2000). Marketing the competitive destination of the future. Tourism Management, 21(1), 97-116.

Casaló, L, Flavián, C, \& Guinalíu, M. (2008). The role of perceived usability, reputation, satisfaction and consumer familiarity on the website loyalty formation process. Computers in Human Behavior, 24(2), 325-345.

Chen, C. F \& Chen, F. S. (2010). Experience quality, perceived value, satisfaction and behavioral intentions for heritage tourists. Tourism Management, 31(1), 29-35.
Chitturi, R, Raghunathan, R, \& Mahajan, V. (2008). Delight by design: the role of hedonic versus utilitarian benefits. Journal of Marketing, 72(3), 48-63.

Chon, K. S. C. \& Olsen, M. D. (1991). Functional and Symbolic Congruity Approaches to Consumer Satisfaction/Dissatisfaction in Consumerism. The Journal of the International Academy of Hospitality Research, 3, 1-25.

Coulter, K. S. \& Coulter, R. A. (2003). The effects of industry knowledge on the development of trust in service relationships. International Journal of Research in Marketing, 20(1), 31-43.

De Rojas, C. \& Camarero, C. (2008). Visitors' Experience, mood and satisfaction in a heritage context: evidence from an interpretation center. Tourism Management, 29(3), 525-537.

Del Bosque, R. I. \& San Martin, H. (2008). Tourist satisfaction a cognitive-affective model. Annals of Tourism Research, 35(2), 551-573.

Deng, S. \& Dart, J. (1994). Measuring market orientation: a multi-factor, multi-item approach. Journal of Marketing Management, 10(8), 725-742.

De Wulf, K, \& Odekerken-Schröder, G. (2003). Assessing the impact of a retailer's relationship efforts on consumer' attitudes and behaviour. Journal of Retailing and Consumer Services, 10(2), 95-108.

Dhar, R. \& Wertenbroch, K. (2000). Consumer choice between hedonic and utilitarian goods. Journal of Marketing Research, 37(1), 60-71.

Ekinci, Y, Dawes, P. L, \& Massey, G. G. (2008). An extended model of the antecedents and consequences of consumer satisfaction for hospitality services. European Journal of Marketing, 42(1-2), 35-68.

Fornell, C. \& Larcker, F. (1981). Structural equation models with unobservable variables and measurement error: algebra and statistics. Journal of Marketing Research, 18(3), 382-388.

Gefen, D. (2000). E-commerce: the role of familiarity and trust. The International Journal of Management, 28(6), 725-737.

Gefen, D, \& Straub, D. W. (2004). Consumer trust in B2C e-Commerce and the importance of social presence: experiments in e-Products and e-Services. The International Journal of Management Science, 32(6), 407-424.

Goodman, L. A. (1960). On the exact variance of products. Journal of the American Statistical Association, 55, 708-713.

Ha, H-Y, \& Perks, H. (2005). Effects of consumer perceptions of brand experience on the web: Brand familiarity, satisfaction and brand trust. Journal of Consumer Behaviour, 4(6), 438-452.

Hair, J. F. Jr, Anderson, R. E, Tatham, R. L, \& Black, W. C. (1998). MULTIVARIATE DATA ANALYSIS, 799 p. 5. ed. Prentice Hall.

Hammitt, W. E, Kyle, G. T, \& Oh, C. O. (2009). Comparison of place models in recreation resource management. Journal of Leisure Research, 41(1), 55-70.

Hirschman, E. C. \& Holbrook, M. B. (1982). Hedonic consumption: emerging concepts, methods and propositions. American Marketing Association, 46(3), 92-101.

Holbrook, M. B. \& Hirschman, E. C. (1982). The experiential aspects of consumption: consumer fantasies, feelings, and fun. Journal of Consumer Research, 9(2), 132-140.

Horng, J. S, Liu, C. H, Chou, H. Y, \& Tsai, C. Y. (2012). Understanding the impact of culinary brand equity and destination familiarity on travel intentions. Tourism Management, 33(4), 815-824.

Hosany, S. \& Gilbert, D. (2010). Measuring tourism emotional experiences toward hedonic holiday destinations. Journal of Travel Research, 49(4), 513-526. 
Hui, T. K, Wan, D, \& Ho, A. (2007). Tourists' satisfaction, recommendation and revisiting Singapore. Tourism Management, 28(4), 965-975.

Jöreskog, K. G. (1971). Simultaneous factor analysis in several populations. Psychometrika, 36(4), 409-426.

Jöreskog, K. G \& Sörbom, D. (1993). New feature in LISREL 8. Chicago: Scientific Software.

Kennedy, M. S, Ferrell, L. K, \& Leclair, D. T. (2001). Consumers' trust of salesperson and manufacturer: an empirical study. Journal of Business Research, 51(1), 73-86.

Kim, J., Forsythe, S., Gu, Q., \& Moon, S. J. (2002). Cross-cultural consumer values, needs and purchase behavior. The Journal of Consumer Marketing, 19(6), 481-502.

Lam, S. Y., Shankar, V., Krishna, E., \& Murthy, B. (2004). Customer value, satisfaction, loyalty, and switching costs: an illustration from a business-to-business service context. Journal of the Academy of Marketing Science, 32(3), 293-311.

Larwood, L., Falbe, C., Kriger, M., \& Miesing, P. (1995). Structure and meaning of organizational vision. Academy of Management Journal, 38(3), 740-769.

Lee, J. S., Lee., C. K., \& Choi, Y. (2011). Examining the role of emotional and functional values in festival evaluation. Journal of Travel Research, $50(6), 685-696$

Lichtenstein, D. R., Netemeyer, R. G., \& Burton S. (1990). Distinguishing coupon proneness from value consciousness: An acquisition - transaction utility theory perspective, Journal of Marketing, 54, 54-67.

Mano, H \& Oliver, R. L. (1993). Assessing the dimensionality and structure of the consumption experience: evaluation, feeling, and satisfaction. Journal of Consumer Research, 20(3), 451-466.

Mathwick, C. H., Malhotra, N., \& Rigdon, E. (2001). Experiential value: conceptualization, measurement and application in the catalog and internet shopping environment. Journal of Retailing, 77(1), 39-56.

Nam, J., Ekinci, Y., Whyatt, W. 2011. Bran Equity, Bran Loyalty and Consumer Satisfaction, Annals of Tourism Research, 38, (3), 1009 - 1030.

O’leary, S., \& Deegan, J. (2005). Ireland's image as a tourism destination in France: attribute importance and performance. Journal of Travel Research, 43(3), 247-256.

Oliver, R. L. (1999). Whence Consumer Loyalty? Journal of Marketing, 63(4), 33-44.

Park, C. (2004). Efficient or enjoyable? Consumer values of eating-out and fast food restaurant consumption in Korea. International Journal of Hospitality Management, 23(1), 87-94.

Pizam, A., \& Ellis T. (1999). Customer satisfaction and its measurement in hospitality enterprises. International Journal of Contemporary Hospitality Management 11(7), 326-339.

Pizam, A., Neumann, Y., \& Reichel, A. (1978). Dimensions of tourist satisfaction with a destination area. Annals of Tourism Research, 5(3), 314322.

Prentice, R. (2004). Tourist familiarity and imagery. Annals of Tourism Research, 31(4), 923-945.

Rogers, H. P., Peyton, R. M., \& Berl, R. L. (1992). Measurement and evaluation of satisfaction processes in a dyadic setting. Journal of Consumer Satisfaction, Dissatisfaction and Complaining Behavior, 5, 12-23.
Servicio Nacional de Turismo. Sernatur. 2008. Recuperado en 01 de marzo, 1999, de http://www.sernatur.cl/.

Sirgy, M. J. (1982). Self-concept in consumer behavioral: a critical review. Journal of Consumer Research, 9(3), 287-300.

Sirgy, M. J., \& Samli, A. C. (1985). A path analytic model of store loyalty involving self-concept, store image, geographic loyalty and socioeconomic status. Journal of the Academy of Marketing Science, 13(3), 265-291.

Sirgy, M. J., \& Su, CH. (2000). Destination image, self-congruity, and travel behavior: toward an integrative model. Journal of Travel Research, 38(4), 340-352.

Snepenger, D., Snepenger, M., Dalbey, M., \& Wessol, A. (2007). Meanings and consumption characteristics of places at a tourism destination. Journal of Travel Research, 45, 310-321.

Sobel, M. E. (1982). Asymptotic intervals for indirect effects in structural equations models. In S. Leinhart (Ed.), Sociological methodology, 290312. San Francisco: Jossey-Bass.

Solomon, M. R. (1983). The role of products as social stimuli: a symbolic interactionism perspective. The Journal of Consumer Research, 10(3), 319-329.

Stedman, R. C. (2002). Toward a social psychology of place predicting behavior from place-based cognitions, attitude, and identity. Environment \& Behavior, 34(5), 561-581.

Stevens, P., Knutson, B., \& Patton, M. (1995). Dineserv: a tool for measuring service quality in restaurants. The Cornell Hotel and Restaurant Administration Quarterly, 36(2), 56-60.

Tsai, S. (2012). Place attachment and tourism marketing: investigating international tourists in Singapore. International Journal of Tourism Research, 14(2), 139-152.

Van der Heijden, H. \& Verhagen, T. (2003). Online store image: conceptual foundations and empirical measurement. Information \& Management, 41(5), 609-617.

Van de Walle, G., Turner, P., \& Davenport, E. (2003). A study of familiarity. In Human-Computer Interaction- INTERACT, 3, 463-70. SVB.

Veryzer, Jr. R.W. (1995). The place of products design and aesthetics in Consumer Research, in Advances in Consumer Research, 22, eds. Frank R. Kardes and Mita Sujan, Provo, UT: Association for Consumer Research, 641-645.

Vieira, V. A. (2008). Moderação, mediação, moderadora-mediadora e efeitos indiretos em modelagem de equações estruturais: uma aplicação no modelo de desconfirmação de expectativas. RAUSP-Revista de Administração da Universidade de Sao Paulo, 44(1), 30-43.

Walmsley, D. J. \& Young, M. (1998). Evaluative images and tourism: the use of personal constructs to describe the structure of destination imag. es. Journal of Travel Research, 36(3), 65-69.

Yoon, Y. \& Uysal, M. (2005). An examination of the effects of motivation and satisfaction on destination loyalty: a structural model. Tourism Management, 26(1), 45-56.

Yüksel, A., Yüksel, F., \& Bilim, Y. (2010). Destination attachment: effects on customer satisfaction and cognitive, affective and conative loyalty. Tourism Management, 31(2), 274-284.

Zaichkowsky, J. L. (1985). Measuring the involvement construct. Journal of Consumer Research, 12, 341-352. 